\title{
急性 $\mathbf{T}$ 淋巴细胞白血病原癌基因 Lmo2 的 一种全新剪接模式 ${ }^{*}$
}

朱天慧 ${ }^{* *}$ 秦 刚

(南开大学医学院, 天津 300071)

\author{
B. Royer-Pokora
}

(Institute of Human Genetics and Anthropology, University of Duesseldorf, 40001 Duesseldorf, Germany)

\begin{abstract}
摘要 Lmo2 是隶属于 LIM 蛋白家族的一个 $\mathrm{T}$ 细胞白血病原癌基因, 研究显示, 它与 胚胎卵黄囊期红系发育、成体血细胞分化相关, 并且在血管新生过程中发挥着重要的作 用. 先前的工作显示该基因编码一个 158 氨基酸的蛋白质, 含有 2 个串联的特征性 LIM 结构域, 但对于其转录调控方式尚缺乏系统的研究. 为进一步了解 Lmo2 转录水平的表 达调控机制, 以正常成人肾组织 mRNA 为材料, 结合 SMART PCR 及 5'RACE 技术找 到了 $L m o 2$ 基因的一种新的剪接模式. 这个新的转录本只含有 2 个外显子, 编码一个 151 氨基酸的蛋白质. 有趣的是, 这个蛋白质与 LMO2 蛋白具有相同的可读框, 只是 $\mathrm{N}$ 端 7 个氨基酸序列不同. 将含有转录起始点上游 294 核苷酸至下游 180 核苷酸的基因组片段 插入报道基因载体并转染 COS7 细胞系, 显示出稳定的启动子活性. 对各种不同来源的 组织、细胞系进行 RT-PCR 检测, 发现此种剪接模式以不同丰度广泛存在.
\end{abstract}

\section{关键词 Lmo2 转录本 剪接模式 启动子活性 RT-PCR}

LIM 蛋白是一个蛋白家族, 其得名缘自该家族中最先发现的 3 个成员 lin-11, isl-1 和 mec-3. LIM 家族成员中都含有 $1 \sim 3$ 个特征性的 52 残基交感序列: (CX2CX17HX2C)-X2-(CX2CX17$\mathrm{CX} 2 \mathrm{C} / \mathrm{H} / \mathrm{D})$, 此序列可形成特征性锌指结构, 称为 LIM 结构域. 目前在不同的真核细胞中已经 发现至少 40 种以上的蛋白质含有 LIM 结构域, 它在进化过程中十分保守, 表明此结构具有非 常重要的功能. 对于 LIM 结构域作用的研究显示, 它介导了 LIM 蛋白二聚化或者 LIM 蛋白与 其他蛋白之间的结合 ${ }^{[1,2]}$, 这拓宽了对于锌指结构生物学功能的认识.

原癌基因 $L m o 2$ (也称 $T t g-2$ 或 Rbtn-2)隶属于 LIM 蛋白家族, 位于人 11 号染色体 $11 \mathrm{p} 13$ 区, 在 $\mathrm{T}$ 细胞白血病中常伴随着此基因上游区段的特异性易位发生 ${ }^{[3,4]}$. 研究发现, 小鼠中 Lmo2 基因与人 $L m o 2$ 具有非常高的同源性, 基因敲除实验已经证实 Lmo2 基因与血细胞生成密切相 关, 并且在血管新生过程中发挥重要作用 ${ }^{[5 \sim 8]}$.

目前对于 $L m o 2$ 转录调控机制的研究不是很多, 有人通过胎肝 cDNA 文库篮选判断此基因 有两种不同的剪接方式, 它们具有不同的转录起始点, 外显子数目也不同, 但编码同一个 158 氨基酸的蛋白质 ${ }^{[9]}$.

为了进一步增进对 Lmo2 转录调控机制的认识, 我们结合 SMART PCR 及 5'RACE 技术,

2000-12-26 收稿, 2001-03-21 收修改稿

* 国家自然科学基金(批准号: 39680024)和天津市自然科学基金(批准号: 993606011)资助项目

** 联系人, E-mail: zhuth@ nankai.edu.cn 
在正常人肾组织中找到了一个全新的 $L m o 2$ 基因剪接方式, 它具有全新的转录起始点, 编码一 个 $\mathrm{N}$ 端与 $\mathrm{LMO} 2$ 不同的含 151 氨基酸的蛋白质. 报道基因检测证实了其调控区具有稳定的启 动子活性, RT-PCR 显示此种剪接模式在不同来源的组织、细胞系中有广泛的表达, 提示其可 能具有基础的生物学功能.

\section{1 材料与方法}

\section{1 实验材料}

正常人肾组织及一急性 $\mathrm{T}$ 淋巴细胞白血病细胞系 8511 的 mRNA, 含有 $L m o 2$ 外显子 5 的 人基因组亚克隆 PUC18-682 为 Institute of Human Genetics and Anthropology, University of Duesseldorf 自制. 胎儿组织及各种细胞系的 mRNA 为本室自制. QuickPrep mRNA 纯化试剂盒 购自 Pharmacia 公司. SMA- RT PCR cDNA 合成试剂盒购自 Clontech 公司. ECL 试剂盒购自 Amersham 公司. Advantage-GC cDNA PCR Kit 购自 Clontech 公司. UNIQ-5 柱离心式 DNA 胶回 收试剂盒购自上海 Sango 生物工程公司. 平端直接克隆试剂盒购自 Novagen 公司. TA 连接试 剂盒购自 Amersham 公司. Lipofection 转染试剂购自 LTI 公司. 苂光素酶活性检测试剂盒购自 TROPIX 公司. $\beta$-半乳糖苷酶检测系统购自 Promega 公司. 各种相关限制性内切酶均购自 Takara 公司. Superscript II 反转录酶及 $\mathrm{T}_{4}$ DNA 连接酶购自 LTI 公司.

\section{2 利用 SMART PCR 反应进行 5'RACE 扩增}

以正常人肾组织 mRNA 为模板, 利用 SMART PCR cDNA 合成试剂盒中的 CDS 为引物, 首先合成 $\mathrm{cDNA}$ 第 1 链, 其中反应体系中加人 $1.7 \mathrm{~mol} / \mathrm{L}$ 的海藻糖至终浓度为 $0.6 \mathrm{~mol} / \mathrm{L}^{[10]}$. 以 SMART 引物和位于第 5 外显子的引物 MMP2(5'-GGAAGTAGCGGTCCCCGATGTTC-3')进行 $\mathrm{PCR}$, 扩增条件为: $95^{\circ} \mathrm{C}$ 预变性 $1 \mathrm{~min}, 94^{\circ} \mathrm{C}, 30 \mathrm{~s}, 68^{\circ} \mathrm{C}, 2 \mathrm{~min}, 25$ 个循环, $68^{\circ} \mathrm{C}$ 延伸 $5 \mathrm{~min}$.

\subsection{Sandwich 杂交}

将 PCR 产物进行 $1.2 \%$ 琼脂糖凝胶电泳, 碱变性, 转移至尼龙膜. 将 Lmo2 cDNA 的可读框 片段用 ECL 试剂盒标记后, 与膜杂交. X 射线片显影, 在约 $400 \mathrm{bp}$ 处得到一条特异性杂交带.

\section{4 片段回收、载体构建及测序}

将杂交呈阳性的 DNA 条带从琼脂糖凝胶回收, 利用 Perfectly Blunt Cloning Kit 将 PCR 产 物连接至 pT7 Blue3 载体上, 用 Li-cor DNA4000 序列仪测序, 得到 439 bp 序列.

\section{5 新剪接模式下的 cDNA 扩增}

在新的 5'端序列中设计引物 Lmo500(5'-GCA GAA GGA GAC CAG AGG TGC-3'), 在原 始 Lmo2 cDNA 序列的 $3^{\prime}$ 末端设计引物 Hop3(5' - TTT ATT AAG CCT GCA GAG CTG-3'), 进 行 PCR 扩增, 条件为: $95^{\circ} \mathrm{C}$ 预变性 $1 \mathrm{~min}, 94^{\circ} \mathrm{C}, 30 \mathrm{~s}, 60^{\circ} \mathrm{C}, 50 \mathrm{~s}, 68^{\circ} \mathrm{C}, 2 \mathrm{~min}, 35$ 个循环, $70^{\circ} \mathrm{C}$ 延伸 $3 \mathrm{~min}$. 得到一个约 $1.6 \mathrm{~kb}$ 片段, 经全自动测序, 推导出全长 $\mathrm{cDNA}$ 序列(图 1), 将此剪接 模式命名为 Lmo2-c . 在 NCBI 的 EST 库中检索时找到一个克隆(Accession Number: H10900), 其剪接模式与我们找到的转录本 $L m o 2-c$ 完全相同, 只是它未到达 $5^{\prime}$ 末端.

\section{6 构建 pGL3-LMO-2np 质粒}

将含有 Lmo2-c 调控区序列的 $474 \mathrm{bp}(-294 \sim+180)$ 片段从基因组亚克隆 puc18-682 切下, 与 苂光素酶报道基因载体 pGL3 连接构建 pGL3-LMO-2np 质粒(图 2). 对插人片段进行全自动测 序, 得到 $5^{\prime}$ 调控区序列(图 1). 将此序列在最近刚刚建立的 NCBI 人类基因组数据库中检索获 
得完全一致的结果.

-291 ctattatgtc ctgatggaat tttgattttg gaaagagaaa gcaacctaa ataatttttg

-231 gtctcattct tcttcttcc tcacctctgc tttgtgcag ttggttaggg ctggagggag (E-box)

-171 gatggcactg gggatttgca gattcttagc actctgggte atctgctgcc ctggcagtga

-111 gcgactctt tagtccagag tgcgtcctt gaggttaggc agtgcccttg ggtgtccagg

-51 ttatgggagg aggaaatga gggagatctg tgaagataga gtcagcgggg agagtgggaa

11 aaagcagaag gagaccagag gtgcccaggt agtgctgggc atgggcaggg gcacagtgcc

71 acctgcaatt gccttgatg cctcttggtg ccctgtgttt acttcttaga catctaggga

131 gtaaggtctg gctctttccc gtcttaaccc aaggttggtt gggactctgc agtctggcca

191 agggatgtgt tgtcctgtcc cttctccett aggtcactga aaggcagtca ggtgagatgc

251 cctgtctggc ccaaaacgat ctcggaccct cggtgctggt ctcactctgg tgaggctgtc

311 cctgatgctt ggccttctct ccagggaacc agtggatgag gtgctgcaga tcccccatc $\begin{array}{llllllllllllllllllllllllllllllllll}M & L & G & L & L & S & R & E & P & V & D & E & V & L & Q & I & P & P & S\end{array}$

371 cctgctgaca tgcggcggct gccagcagaa catcggggac cgctacttcc tgaaggccat L I T G G G G

431 cgaccagtac tggcacgagg actgcetgag ctgcgacctc tgtggctgcc ggctgggtga D o y

491 ggtggggcgg cgcctctact acaaactggg ccggaagctc tgccggagag actatctcag

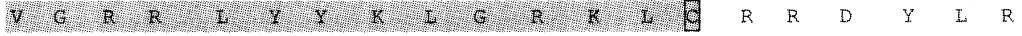

551 gcttttggg caagacggtc tctgcgcatc ctgtgacaag cggattcgtg cotatgagat

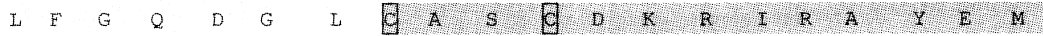

611 gacatgcgg gtgaaagaca aagtgtatca cctggaatgt ttcaagtgcg ccgcctgtca

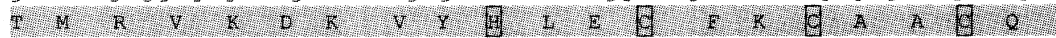

671 gaagcattc tgtgtaggtg acagatacct cctcatcaac tctgacatag tgtgcgaaca

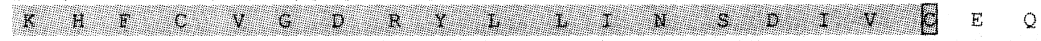

731 ggacatctac gagtggacta agatcaatgg gatgatatag gcccgagtcc cogggcatct $D \quad I \quad Y \quad E \quad W \quad T \quad K \quad I N \quad N \quad G \quad M \quad I \quad *$

791 ttggggaggt gttcactgaa gacgccgtct ccatggcatc ttcgtcttca ctcttaggca

851 ctt

911 atcaagacat agcatccaag tggcataatt caggggctga cacttcaagg tgacagaagg

971 accagccctt gagggagaac ttatggccac agcccatcca tagtaactga catgattagc

1031 agaagaaagg aacatttagg ggcaagcagg cgctgtgcta tcatgatgga atttcatatc

1091 tacagataga gagttgttgt gtacagactt gttgtgactt tgacgcttgc gaactagaga

1151 tgtgcaattg atttctttc ttcctggct tttaactccc ctgttcaat cactgtcctc

1211 cacacaaggg aaggacagaa aggagagtgg ccattcttt tttcttggcc cccttcccaa

1271 ggcctaagc tttggaccca agggaaact gcatggagac gcatttcggt tgagaatgga

1331 aaccacaact tttaaccaaa caattattta aagcaatgct gatgaatcac tgttttaga

1391 cacctcatt ttgaggggag gagttccaca gattgtttct atacaaatat aaatcttaaa

1451 aggttgttca actattttat tatcctagat tatatcaaag tatttgtcgt gtgtagaaaa

1511 aaaaacagc tctgcaggct taataaaat gacagactga aaaaaaaaa a

图 $1 \quad L m o 2-c$ 全长 cDNA 及调控区序列

\section{7 细胞培养}

COS7 细胞系在含有 $10 \%$ 胎牛血清的 DMEM 培养基中贴壁生长. HEL 及 HPB 细胞系在含 有 $10 \%$ 胎牛血清的 RPMI-1640 培养基中悬浮培养.

\section{8 转染 COS7 细胞系, 检测荧光素酶的活性}

将 pGL3-LMO-2np, pGL3-Basic 分别与 pSV- $\beta$-Gal 对照载体共转染 COS7 细胞, 然后分别 测定苂光素酶和 $\beta-G a l$ 的活性. 以 $\beta-G a l$ 活性值校正后的“相对苂光素酶活性”来表示报道基因 的表达活性. 具体方法依照使用说明书进行, 独立进行 3 次实验.

\section{9 各种不同来源组织、细胞系的 RT-PCR 分析}

利用 mRNA 纯化试剂盒纯化胎脑、肝、胸腺、肾、成人肝以及 HEL, HPB 细胞系的 mRNA, 按照标准方法进行反转录. 在 Lmo2-c 的 cDNA 序列中设计引物 LMO2-X(5'-ATGCTTGGCCTTCTCTCC-3'), LMO2-r1 (5'-CCTATATCATCCCATTGATC-3')进行 PCR, 扩增条件为: $95^{\circ} \mathrm{C}$ 预 
变性 $1 \mathrm{~min}, 94^{\circ} \mathrm{C}, 30 \mathrm{~s}, 58^{\circ} \mathrm{C}, 50 \mathrm{~s}, 68^{\circ} \mathrm{C}, 2 \mathrm{~min}, 35$ 个循环, $70^{\circ} \mathrm{C}$ 延伸 $3 \mathrm{~min}$. 选用原始 $\mathrm{Lmo} 2$ 基因 cDNA 序列中的引物 MR10 (5' -CGAATCCGCTTGTCACAGGA-3'), MR12(5' -GCCATCGAAAGGAAGAGCC-3')进行 PCR, 扩增条件为: $95^{\circ} \mathrm{C}$ 预变性 $1 \mathrm{~min}, 94^{\circ} \mathrm{C}, 30 \mathrm{~s}, 60^{\circ} \mathrm{C}, 50 \mathrm{~s}$, $68^{\circ} \mathrm{C}, 2 \mathrm{~min}, 35$ 个循环, $70^{\circ} \mathrm{C}$ 延伸 $3 \mathrm{~min}$. 以 $\beta$-actin 作为内参照, 扩增条件为: $95^{\circ} \mathrm{C}$ 预变性 $1 \mathrm{~min}$, $94^{\circ} \mathrm{C}, 30 \mathrm{~s}, 60^{\circ} \mathrm{C}, 50 \mathrm{~s}, 68^{\circ} \mathrm{C}, 2 \mathrm{~min}, 35$ 个循环, $70^{\circ} \mathrm{C}$ 延伸 $3 \mathrm{~min}$.

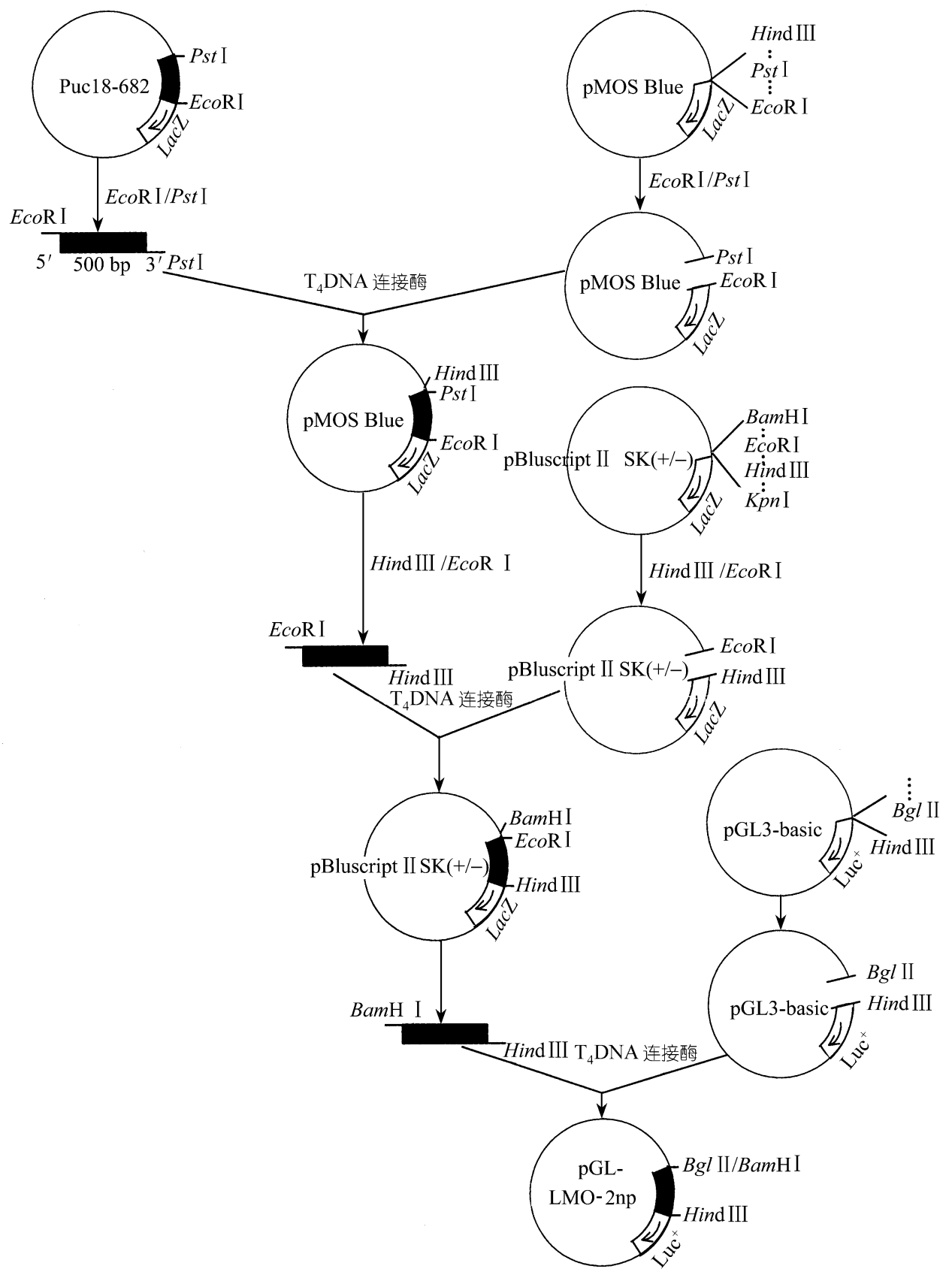

图 2 重组质粒 pGL3-LMO-2np 构建示意图 


\section{2 结果}

\section{$2.1 L m o 2$ 基因具有另外一种剪接方式}

采用基于 SMART PCR 技术的 5'RACE 方法，在原始 Lmo2 基因编码区内设计引物 MMP2, 以正常成人肾组织 mRNA 为材料扩增到 Lmo2 基因的一个新的剪接模式, 其全长为 1561 $b p$ (GenBank 登记号: AF257211). 这个新剪接模式的 $5^{\prime}$ 端与原先从胎肝 cDNA 文库中克隆到的 Lmo2 不同, 仅由 2 个外显子组成, 新的外显子 1(553 bp) 是在原来的外显子 5(217bp)的基础上 又向 5 端，即原来内含子 4 的部位延伸了 $336 \mathrm{bp}$. 外显子 2 则与原来的外显子 6 完全相同. 为 了区别 $L m o 2$ 原有的剪接模式 $L m o 2-a / b$, 我们称这种新的剪接模式为 Lmo2-c. Lmo2-c 具有与原 始 Lmo2 完全相同的可读框, 都含特征性的 LIM 结构域, 但 LMO2-c 蛋白比 LMO2 少 7 个氨基 酸，且 $\mathrm{N}$ 末端 7 个氨基酸序列不同(LMO2 的 3 种不同剪接方式见图 3).

(a)

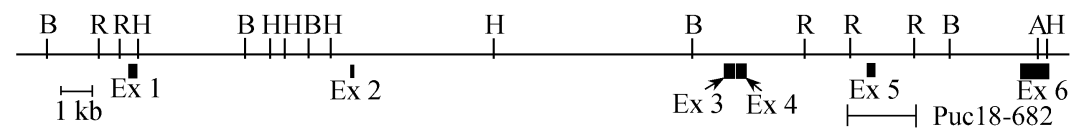

(b) Lmo2-a

Ex 1a

$\operatorname{Ex} 2 \mathrm{a}$

Lmo2-b

Ex 3aEx 4a Ex 5a Ex 6a

Lmo2-c

\begin{tabular}{ccccc}
\hline & 外显子数 & mRNA/bp & 编码区/bp & 蛋白质/AA \\
\hline Lmo2- $a$ & 6 & 2056 & 474 & 158 \\
Lmo2- $b$ & 4 & 1744 & 474 & 158 \\
Lmo2-c & 2 & 1561 & 453 & 151 \\
\hline
\end{tabular}

图 $34 m o 2$ 基因的 3 种不同剪接方式

(a) Lmo2 基因的基因组限制性内切酶图谱, Puc18-682 显示 $E c o \mathrm{R} \mathrm{I} 2.2 \mathrm{~kb}$ 基因组片段所在位置（B 示 BamH I ; $\mathrm{H}$ 示 Hind III ; R 示 EcoR I ; A 示 Acc I ); (b) 3 种不同剪接方式和编码产物

\section{$2.2 \quad L m o 2-c 5^{\prime}$ 端相邻调控区序列具有启动子活性}

为了验证 $L m o 2-c$ 的启动子活性，将含有相应调控区域的基因组片段(-294 +180)接到报 道基因载体 pGL3-basic 上, 并将构建的 pGL3-LMO-2np 质粒和 pGL3-basic 分别与 $\beta$-半乳糖苗 酶检测载体共转染 COS7 细胞, 经过 $\beta$-半乳糖苷酶活性校正后, 以 pGL3-basic 转染 COS7 细 胞的苂光素酶表达活性为 1 , 则 pGL3-LMO-2np 的相对苂光素酶表达活性为 4.5. 对该启动子 区域测序, 并进行了转录调控元件的分析, 没有发现普遍存在的 TATA 框结构, 但存在典型的 GATA 位点以及 $\mathrm{E}$ 框(图 1).

\section{$2.3 \mathrm{Lmo2-c}$ 以及 $\mathrm{Lmo2}$ 在各种组织、细胞系中的表达谱分析}

选择 $L m o 2$ 基因新老剪接转录本的特异性区段设计引物进行 RT-PCR，检测了它们在不同 组织、细胞系中的表达情况 (图 4). 结果显示, 在急性 $\mathrm{T}$ 淋巴细胞白血病细胞系 8511 中两者皆 有高量表达; 在另一 $\mathrm{T}$ 急性淋巴细胞系 HPB 中看不出 Lmo2(包括 Lmo2-a/Lmo2-b) 表达, 而 Lmo2-c 则有高量表达; 在红白血病细胞系 HEL 中 Lmo2 表达量很高, 而 Lmo2-c 表达则较少; Lmo2 在胎脑、胎肝中表达量很高, 在胎肾及成人肝中表达量适中, 但在胎儿胸腺中表达量很 
低; Lmo2-c 在胎肝及成人肝中都具有较高水 平表达, 但在胎脑、胎肾、胎儿胸腺中表达 水平都不高.

\section{3 讨论}

在各种不同类型的急性白血病中, 染色 体的易位是非常普遍的, 研究发现这些易位 发生的位点绝非随机, 而是主要定位于各种 转录调节因子; 染色体由于发生易位而改变 了特定转录因子基因表达调控区或编码区 的结构, 进而干扰了正常的细胞增殖、分化 以及调亡的进行 ${ }^{[11]}$. Lmo2 在正常 $\mathrm{T}$ 淋巴细胞 中是不表达的, 但在大部分染色体 11p13 区 异常 ( 例如 发生 $\mathrm{t}(11 ; 14)(\mathrm{p} 13 ; \mathrm{q} 11)$ 或 $\mathrm{t}(7 ; 11)(\mathrm{q} 35 ; \mathrm{p} 13)$ 易位)的急性 $\mathrm{T}$ 淋巴细胞白 血病中有过量表达 ${ }^{[4,12]}$, 显示它与 TAL1 一

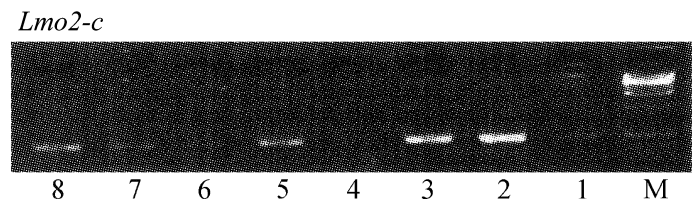

Lmo2

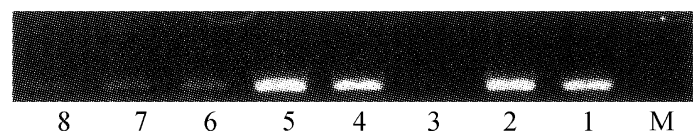

$\beta$-actin

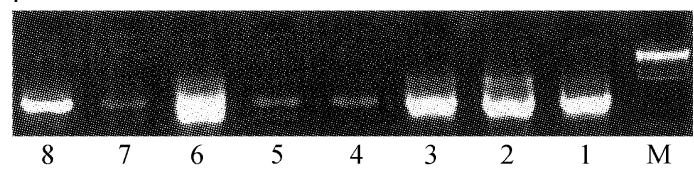

图 $4 L m o 2$ 新老剪接模式以及 $\beta$-actin 在不同胎儿、 细胞系的 RT-PCR 分析结果

M 示 marker, 1 示 HEL, 2 示 8511,3 示 HPB, 4 示胎脑, 5 示胎 肝, 6 示胎儿胸腺, 7 示胎肾, 8 示成人肝 样可能在白血病发生过程中发挥着重要作用 ${ }^{[5,13 ~ 15]}$. 对于 LMO2 作用的分子机制已见很多报 道, 结果显示, 它是一种重要的转录调控因子, 但与众不同的是 LMO2 自身不具备结合 DNA 的结构域, 其特征性的 LIM 结构域可介导蛋白质之间的结合, 与其他转录调控因子如 SCL(TAL1), GATA-1，LDB1，RBP2 等相互作用形成各种不同类型的复合体，进而调控特定的 下游靶基因的表达 ${ }^{[16 ~ 21]}$ 。对小鼠的基因敲除实验发现, Lmo2 在胚胎卵黄囊期红系发育、血细 胞分化以及血管新生等生物过程中都起着不可或缺的作用 ${ }^{[5 \sim 8]}$. 目前 $L m o 2$ 在 $\mathrm{T}$ 淋巴细胞白血 病中异常表达的机制尚不清楚，它在白血病发生中所起的作用也处于推测阶段 ${ }^{[22,23]}$.

本实验结合 SMART PCR 及 5'RACE 技术成功地在成人肾组织中找到了原癌基因 Lmo2 的一种新的剪接模式, 命名为 Lmo2-c. LMO2-c 编码一个与 LMO2 的 $\mathrm{N}$ 端序列不同的蛋白质, 比 LMO2 少 7 个氨基酸, 但是在保守的 LIM 结构域区域没有任何变化. 为了进一步确证这个 新的转录起始点, 我们构建了含有 Lmo2-c 基因 $5^{\prime}$ 端转录调控序列的苂光素酶报道基因质粒 pGL3-LMO-2np, 并转染到 COS7 细胞中, 经报道基因活性检测证实此序列确实具有稳定的启 动子活性. 对启动子区域序列进行分析, 没有找到特征性的的 TATA 框及 CCAAT 框, 但找到 了典型的 GATA 位点和 E 框, 这些特点与先前得到的 Lmo2 启动区 P1 具有惊人的相似性 ${ }^{[9]}$, 推 测这两种剪接模式的转录调控机制很接近.

研究发现, LMO 蛋白序列是高度保守的, 人与小鼠的 LMO1 蛋白序列中只有两个编码氨 基酸不同, LMO2 中只有一个氨基酸不同，而对于 LMO3 序列则完全相同 ${ }^{[24]}$. 虽然这些基因在 人和小鼠中分别定位于不同染色体, 但每一个基因在人和鼠中都表现为相似的外显子组织结 构. 以往认为 Lmo2 基因只存在一种蛋白编码产物, 对其功能及分子作用机制的研究也都建立 在这种观点的基础上. 我们从人的正常组织中克隆到了 Lmo2 的另一种 mRNA 剪接模式, 编码 一个 $\mathrm{N}$ 端不同的截短蛋白质. 有趣的是这个蛋白质保留了特征性的 LIM 结构域, 而先前的研 究显示正是 LIM 结构域在介导 LMO2 与其他蛋白相互作用中发挥着直接作用. 这一特点使我 
们联想到另一个急性 $\mathrm{T}$ 淋巴细胞白血病相关癌基因 Tall, 它具有全长、截短两种蛋白产物, 且 都含有特征性的 bHLH 结构域 ${ }^{[25,26]}$. 由于先前的小鼠 Lmo2 基因敲除试验都是定位在第 1 个 LIM 结构域 ${ }^{[5 ~ 8]}$, 而我们新发现的 LMO2 截短形式同样含有这个区域, 所以有理由质疑 Lmo2 在血细胞生成、血管新生、白血病发生等过程中发挥的作用是否由单一的 LMO2 蛋白行使. 虽 然目前我们还未能确证这种截短的 LMO2 蛋白也存在于小鼠中, 但比较人鼠 Lmo1 及 Lmo3 序 列同源性的证据强烈支持这个假设 ${ }^{[24]}$, 这也正是我们下一步要证实的问题.

总之, 我们利用近期发展出来的 cDNA $5^{\prime}$ 端克隆技术, 从人组织中克隆到急性白血病原 癌基因 Lmo2 的一种新的剪接模式, 它编码一个 $\mathrm{N}$ 端序列不同但保留了特征性 LIM 结构域的 蛋白质. 此种剪接模式的发现，增进了我们对于 Lmo2 基因表达调控方式的认识，为全面认识 Lmo2 的生物学功能提供了重要线索.

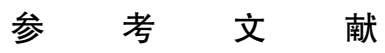

1 Feuerstein R, Wang X, Song D, et al. The LIM/double zinc-finger motif functions as a protein dimerization domain. Proc Natl Acad Sci USA, 1994, 91: 10655 10659

2 Schmeichel K L, Beckerle M C. The LIM domain is a modular protein-binding interface. Cell, 1994, 79: $211 \sim 219$

3 Boehm T, Foroni L, Kaneko Y, et al. The rhombotin family of cystein-rich LIM-domain oncogenes: distinct members are involved in T-cell translocations to human chromosome 11p15 and 11p13. Pro Natl Acad Sci USA, 1991, 88: 4367 4371

Royer-Pokora B, Loos Uschi, Ludwig W D, et al. TTG-2, a new gene encoding a cysteine-rich protein with LIM motif, is overexpressed in acute T-cell leukaemia with the $t(11 ; 14)(\mathrm{p} 13 ; \mathrm{q} 11)$. Oncogene, 1991, 6: $1887 \sim 1893$

5 Warren A J, Colledge W H, Carlton M B, et al. The oncogenic cysteine-rich LIM domain protein Rbtn2 is essential for erythroid development. Cell, 1994, 78: $45 \sim 57$

6 Visvader J E, Mao X H, Fujuwara Y, et al. The LIM-domain binding protein Ldb1 and its partner LMO2 act as negative regulators of erythroid differentiation. Pro Natl Acad Sci USA, 1997, 94: $13707 \sim 13712$

7 Yamada Y, Warren A J, Dobson C, et al. The T cell leukemia LIM protein Lmo2 is necessary for adult mouse hematopoiesis. Pro Natl Acad Sci USA, 1998, 95: $3890 \sim 3895$

8 Yamada Y, Pannell R, Forster Alan, et al. The oncogenic LIM-only transcription factor Lmo2 regulates angiogenesis but not vasculogenesis in mice. Proc Natl Acad Sci USA, 2000, 97: $320 \sim 324$

9 Royer-Pokora B, Rogers M, Zhu T H, et al. The TTG-2/RBTN-2 T cell oncogene encodes two alternative transcripts from two promoters: the distal promoter is removed by most $11 \mathrm{p} 13$ translocations in acute T cell leukaemia's (T-ALL). Oncogene, 1995, 10: $1353 \sim 1360$

10 Carninci P, Nishiyama Y, Westover A, et al. Thermostabilization and thermo-activation of thermolabile enzymes by trehalose and its application for the synthesis of full length cDNA. Proc Natl Acad Sci USA, 1998, 95(2): $520 \sim 524$

11 Look A T. Oncogenic transcription factors in the human acute leukemias. Science, 1997, 278: 1059 1064

12 Fitzgerald T J, Neale G A M, Raimondi S C, et al. Rhom-2 expression does not always correlate with abnormalities on chromosome 11 at band p13 in T-cell acute lymphoblastic leukemia. Blood, 1992, 80: $3189 \sim 3197$

13 Robb L, Lyons I, Li R, et al. Absence of yolk sac hematopoiesis from mice with a targeted disruption of the scl gene. Proc Natl Acad Sci USA, 1995, 92: $7075 \sim 7079$

14 Shivdasani R A, Mayer E L, Orkin S H. Absence of blood formation in mice lacking the T-cell leukaemia oncoprotein tal-1/SCL. Nature, 1995, 373: $432 \sim 434$ Aplan P D, Jones C A, Chervinsky D S, et al. An scl gene product lacking the transactivation domain induces bony abnormalities and cooperates with LMO1 to generate T-cell malignancies in transgenic mice. EMBO J, 1997, 16: $2408 \sim 2419$ Valge-Archer V E, Osada H, Warren A J, et al. The LIM protein RBTN2 and the basic helix-loop-helix protein TAL1 are 
present in a complex in erythroid cells. Pro Natl Acad Sci USA, 1994, 91: 8617 8621

17 Wadman I, Li J, Bash R, et al. Specific in vivo association between the bHLH and LIM proteins implicated in human T cell leukemia. EMBO J, 1994, 13: 4831 4839

18 Wadman I A, Osada H, Grutz G G, et al. The LIM-only protein Lmo2 is a bridging molecule assembling an erythroid, DNA-binding complex which includes the TAL1, E47, GATA1 and Ldb1/NLI proteins. EMBO J, 1997, 16: $3145 \sim 3157$

19 Visvader J E, Mao X H, Fujiwara Y, et al. The LIM-domain binding protein Ldb1 and its partner LMO2 act as negative regulators of erythroid differentiation. Proc Natl Acad Sci USA, 1997, 94: 13707 13712

20 周 严, 杜光伟, 汪俊华, 等. 人 LDB1 cDNA 的分离与克隆. 科学通报, 1999, 44: 755 760

21 Grutz G G, Bucher K, Lavenir I, et al. The oncogenic T cell LIM-protein Lmo2 forms part of a DNA-binding complex specifically in immature T cells. EMBO J, 1998, 17: 4594 4605

22 Rabbitts T H. LMO T-cell translocation oncogenes typify genes activated by chromosomal translocations that alter transcription and developmental processes. Gene \& Dev, 1998, 12: $2651 \sim 2657$

23 Rabbitts T H, Bucher K, Chung G, et al. The effect of chromosomal translocations in acute leukemias: The LMO2 paradigm in transcription and development. Cancer Res, 1999, 39: $1794 \sim 1798$

24 Foroni L, Boehm T, White L, et al. The rhombotin gene family encode related LIM-domain proteins whose differing expression suggests multiple roles on mouse development. J Mol Biol, 1992, 226: 747 761

25 Bernard O, Lecointe N, Jonveaux P, et al. Two site-specific deletions and $\mathrm{t}(1 ; 14)$ translocation restricted to human T-cell acute leukemias disrupt the 5' part of the tal-1 gene. Oncogene, 1991, 6: $1477 \sim 1488$

26 Cheng J T, Hsu H L, Hwang L Y, et al. Products of the TAL1 oncogene: basic helix-loop-helix proteins phosphorylated at serine residues. Oncogene, 1993, 8: $677 \sim 683$ 